\title{
Is it relevant a correct intimate cleansing in the treatment of vulvar dermatosis?
}

\author{
Filippo Murina, Stefania Di Francesco, Martina Ratti
}

Department of Vulvar Disease, V. Buzzi Hospital, University of Milan, Milan, Italy

Email: filippomurina@tin.it

Received 27 December 2013; revised 25 January 2014; accepted 3 February 2014

Copyright (C) 2014 Filippo Murina et al. This is an open access article distributed under the Creative Commons Attribution License, which permits unrestricted use, distribution, and reproduction in any medium, provided the original work is properly cited. In accordance of the Creative Commons Attribution License all Copyrights (c) 2014 are reserved for SCIRP and the owner of the intellectual property Filippo Murina et al. All Copyright (C) 2014 are guarded by law and by SCIRP as a guardian.

\section{ABSTRACT}

Background: Vulvar dermatoses are inflammatory disorders of genital skin causing itch, pain, dysuria and dyspareunia. Objective: To compare the efficacy of two intimate cleansers as adjuvants in the treatment of the two common vulvar dermatoses: Lichen sclerosus (LS) and lichen simplex chronicus (LSC). Method: 32 patients with the diagnosis of LS or LSC initially treated with Mometasone furoate $0.1 \%$ cream were then randomly assigned to receive either cleanser A (cleaner based on amigdalus dulcis, malva, jojoba oil, PCL solid, Hyaluronic acid) or cleanser B (cleaner based on Cocoyl Wtheat Amino Acids, Maltodextrins, Aveena sativa, Caprylic Glycol) twice a day in association with lanolin cream one application daily for 30 days, following which the lanolin cream was applied only when needed as a rescue treatment. The amount of the rescue cream used, rubbing, applicability and pleasantness of the product were classified before and after 60 days of treatment. Results: The differences between the two treatments for rubbing, applicability and pleasantness of the product were statistically non-significant, while "as needed" use of the emollient lanolin cream was significantly superior in a total number of doses in the $B$ group versus the $A$ group (8.25 vs 5.28 (P < 0.001). Conclusion: The parameters showed a high score for both the cleansers, demonstrating the acceptability of these products by the women. The best results of the cleaner $A$ can be attributed to not only to the pool of moisturizing and emollient active ingredients but that of the soothing effects as well. This shows that the role of intimate cleanser is not only for vulval cleansing, but can also play a therapeutic role of moisturization and emollience.

\section{KEYWORDS}

Vular Dermatosis; Lichen Sclerosus; Lichen Simplex Chronicus; Intimate Cleansers

\section{INTRODUCTION}

Vulvar dermatoses are inflammatory muco-cutaneous disorder of genital skin that form a frequent finding in the specialized center for vulvar diseases. They are debilitating diseases that cause itching, pain, dysuria, dyspareunia, and significant sexual dysfunction in women. Most commonly encountered vulvar dermatoses are Lichen sclerosus (LS) and lichen simplex chronicus (LSC) [1]. LS is a chronic, inflammatory skin disease with a distinct predilection for the anogenital region [2]. The exact prevalence of LS is difficult to ascertain and probably underestimated, since patients with LS may present to various clinical specialties or physicians who might not always recognize LS and probably because the patients are asymptomatic [3]. Many findings point more and more towards an autoimmune-induced disease in genetically predisposed patients [4]. The diagnosis of LS is usually clinical. However, in the early stages of the disease the diagnosis can be difficult and in these cases a histological examination is required [5]. LSC is caused by persistent itching and scratching of vulvar skin, resulting in a thickened leathery appearance. It is thought to be an atopic disorder in many cases and may also arise in normal skin [6]. In both the disorders, the area involved may vary from a single small to the entire region of vulva, perineum, and perianus [7]. The primary lesions of vulvar dermatosis are white/red, flat and/or thickened areas. Edema may be prominent. Fragility is a hallmark of LS, manisfecting as erosions, fissuring, purpura, and ecchymoses. Fissuring is especially common between the clitoris, urethra, in the interlabial sulci, and at the base of 
the posterior fourchette [7]. The typical lesions are porcelain-white papules and plaques with follicular delling and hyperkeratosis. The area evolves into a dry, hypopigmented, sclerotic, and later atrophic lesion. The clinical and histological alterations of vulvar dermatoses are responsible for changes in vulvar skin barrier function that enhances the symptoms (dehydration and impaired lipid skin film). According to the British Association of Dermatologists guidelines the treatment of both LS and LSC should begin with corticosteroid ointments or creams of varying potency [8]. It is also recommended to use emollient creams with the aim of restoring the protective skin barrier and the use of products for personal hygiene. The intimate cleansers should contain the appropriate active ingredients with proven efficacy, and in particular must not contain surfactants that are aggressive or have tendency to induce genital dryness. They must also contribute to the maintenance or restoration of the physiological protective lipid film, through the association of their moisturizing and emollient properties. The objective of this study was to compare the efficacy of two intimate cleansers of reference as adjuvants in the treatment of vulvar dermatoses.

\section{MATERIALS AND METHODS}

The experimental design was a controlled, randomized, clinical study in which 32 patients with vulvar dermatoses were recruited. The diagnosis was either of LS or LSC. Each patient received Mometasone furoate in the form of $0.1 \%$ cream. The treatment was intensive during the first month. It consisted of the use of $1 / 2$ fingertip unit (FTU), i.e. the amount of topical Mometasone furoate that is squeezed out from a standard tube on the fingertip of an adult (that is about $0.5 \mathrm{~g}$ of topical steroid) once a day for four weeks, then twice a week for four more weeks and subsequently topical steroid was applied only when needed as a rescue treatment. We then divided the patients in two Groups according to the randomization list: Group A: the patients were prescribed to use the cleanser A twice a day in association with one application of lanolin cream daily for 30 days, following which the lanolin cream was applied only when needed as a rescue treatment. The cleaner A was a non-irritant cleaner based on specific substances with cleansing, hydrating, antimicrobial and anti-inflammatory properties (amigdalus dulcis, malva, jojoba oil, PCL solid, Hyaluronic acid). Group B: the patients were prescribed to use the cleanser $\mathrm{B}$ in association with one application of lanolin cream daily for 30 days, and after that the lanolin cream was applied only when needed as a rescue treatment. The cleaner B was a non-irritant cleaner based on soft natural surfactants (Cocoyl Wtheat Amino Acids) and with hydrating and emollient substances (Maltodextrins, Aveena sativa, Caprylic Glycol).
The parameters evaluated for efficacy were the following: amount of the rescue cream used, rubbing, applicability and pleasantness of the product. Each descriptor was ranked on a 4 -point intensity scale $(0=$ none, $1=$ mild, 2 = moderate and $3=$ severe). The signs and symptoms were classified before and after 60 days of treatment. A statistical analysis for the comparison of the two treatments was carried out by applying the chisquare test (improved vs impaired/unchanged versus baseline) and Student's t test (amount of rescue cream employed).

\section{RESULTS}

The characteristics of the study population are summarized in Table 1. The women in the two groups were similar for age (52 years, range 32 - 73 for cleanser A vs 56 years, range 34 - 69 for cleanser B) and diagnosis.

The differences between the two treatments for rubbing, applicability and pleasantness of the product were statistically non-significant (Figure 1).

The dryness was quantified indirectly through the average number of applications of Lanolin cream as a rescue treatment. The "as needed" use of the emollient lanolin cream was significantly superior in a total number of doses in the B group versus the A group (8.25 vs 5.28 $(\mathrm{P}<0.001)$.

\section{DISCUSSION}

A Vulvar dermatoses are mainly treated with topical ste-

Table 1. Characteristics of the study populations.

\begin{tabular}{cccc}
\hline & Group A & Group B & P value \\
\hline Number of cases & 16 & 16 & \\
Age (years, mean and range) & $52(32-73)$ & $56(34-69)$ & NS \\
Diagnosis (numbers) & 12 LS-4 LSC & 13LS-3 LSC & NS \\
\hline
\end{tabular}

LS = lichen sclerosus; LSC = lichen symplex.

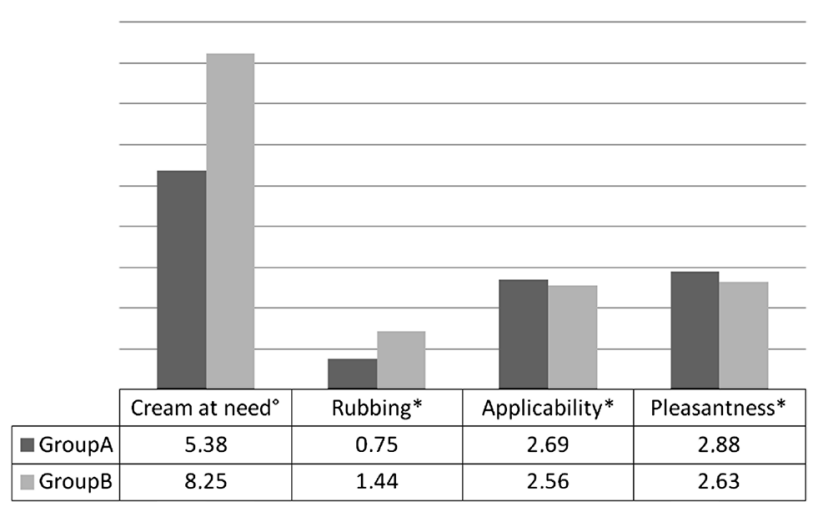

Figure 1. Symptoms after treatment. ${ }^{\circ}$ Days; * Score 0 - $3(0=$ none, 1 = mild, 2 = moderate and 3 = severe), means $\mathrm{P}$ value: cream at need $=0.001$, rubbing $=0.05$, applicability $=0.03$ pleasentness $=0.06$. 
roids to relieve the symptoms, prevent structural damages and to reverse histologic changes. The recommended regimen for LS and LSC, the most frequent vulvar dermatosis, begins with a high-potency corticosteroid used daily until the resolution of active lesions after which it is tapered to once or twice per week [8]. A gradual dose reduction enables the majority of patients to remain in asymptomatic remission and minimizes the steroid exposure [9].

In vulvar dermatosis problems related to the damage of the barrier function of vulvar skin can arise. The skin barrier depends on the degree of hydration, the presence of a horny/corneum layer and an intact surface. The surface lipid film is the main vulvar skin protective factor. It consists of a hydrophilic component (Natural Moisturizing Factor = NMF) and a soluble fraction, mainly consisting of sebum. Treatment of vulvar dermatoses should be aimed to restore the lipid film surface to protect the vulvar skin which requires the need of using emollient preparations in addition to topical steroid therapy and specific products for personal hygiene [10].

In our study we compared two different cleaners as adjuvants in the treatment of vulvar dermatoses. The parameters, namely applicability and acceptability/pleasantness, showed a high score for both the cleansers, demonstrating the acceptability of these products by the women and hence, an increase in the compliance.

Both cleansers show a strong activity in limiting the vulvar rubbing (cleaner A versus cleaner B, 0.75 versus 1.44, $\mathrm{p}=0.02$ ); even though the higher activity which occured with cleaner A had a borderline significance, but this difference in favor of the cleaner A is clinically important, as it was achieved despite the $\mathrm{B}$ cleaner group applied a significantly higher number of doses of emollient cream as a rescue treatment. The rubbing is an indirect index of friction and that of the superficial dryness. This emphasizes the moisturizing properties of the two products. The number of days required for the application of the moisturizing cream on demand, is the most different parameter in the two groups of patients ( $\mathrm{A}=$ 5.38 days; $\mathrm{B}=8: 25$ days) with an average that is statistically significant $(\mathrm{p}=0.001)$, and in any case low for both groups.

The best results of the cleaner A can be attributed to not only the pool of moisturizing and emollient active ingredients but that of the soothing as well.

This shows how the role of intimate cleanser is not only for the cleansing of vulva, but can also play a therapeutic role of moisturization and emollience. This is aimed at restoration of the skin surface lipid film, compromised in vulvar dermatoses where the interruption of skin barrier effect increases dehydration and promotes the onset of rigidity and superficial erosions, supporting the persistence of vulvar itching and burning. This ap- pears even more evident if the cleanser has specific active ingredients distinctly moisturizing, soothing and decongestant (Cleaner A).

In order to soothe, clean and protect the external mucosa in an eudermic fashion the cleanser should not contain alkaline and occlusive substances as well as anionic surfactants. It should clean by affinity and contain ingredients that are complementary and synergic.

Specific active ingredients of Cleaner A are: Amygdalus dulcis that contains high percentages of fatty acids with special eurdermic properties:jojoba oil, that is an anti-inflammatory and emollient that stimulates the cutaneous circulation; the Hyaluronic acid that is able to incorporate large quantities of water maintaining the degree of moisturization, turgidity, plasticity and viscosity. Hyaluronic acid acts like a cementing, anti-collision substance as well as an efficient lubrificant thus, preventing the damage of tissue by the physical stress. It also acts like a filter against the free diffusion of certain substances, bacteria and infecting agents in the tissue; European aloe: the unsaponifiable fraction strongly attracted to the skin sebum performs protetive and sebum substituting functions. It acts on the skin trophism stimulating the repairing processes in the derma and epidermis; the solid PCL, another active ingredient of cleaner A, has significant moisturizing and film-forming properties, that are particularly active in recreating the protective film surface.

\section{CONCLUSION}

This new-generation intimate cleanser was effective in reducing burning and providing a concomitant emollience in the treatment of vulvar dermatoses. In particular, the product acts by "affinity" and not by "contrast" like the most surfactant based foaming cleansers. Again we stress the need for a proper hygiene with selected products that meet the vulvar skin requirements.

\section{REFERENCES}

[1] Powell, J.J. and Wojnarowska, F. (1999) Lichen sclerosus. Lancet, 353, 1777-1783. http://dx.doi.org/10.1016/S0140-6736(98)08228-2

[2] Murphy, R. (2010) Lichen sclerosus. Dermatologic Clinics, 28, 707-715. http://dx.doi.org/10.1016/j.det.2010.07.006

[3] Goldstein, A.T., Marinoff, S.C., Christopher, K., et al. (2005) Prevalence of vulvar lichen sclerosus in a general gy-necology practice. The Journal of Reproductive Medicine, 50, 477-480.

[4] Cooper, S.M., Ali, I., Baldo, M., et al. (2008) The association of lichen sclerosus and erosive lichen planus of the vulva with autoimmune disease: A case-control study. Archives of Dermatology, 144, 1432-1435. http://dx.doi.org/10.1001/archderm.144.11.1432 
[5] Regauer, S., Liegl, B., Reich, H., et al. (2004) Vulvar lichen sclerosus: The importance of early clinical and histo-logical diagnosis. Hautarzt, 55, 158-164. http://dx.doi.org/10.1007/s00105-003-0645-8

[6] Lynch, P.J. (2004) Lichen simplex chronicus (atopic/ neurodermatitis) of the anogenital region. Dermatology and Therapy, 17, 8-19.

http://dx.doi.org/10.1111/j.1396-0296.2004.04002.x

[7] Burrows, L.J., Shaw, H.A. and Goldstein, A.T. (2008) The vulvar dermatoses. The Journal of Sexual Medicine, 5, 276-283.

http://dx.doi.org/10.1111/j.1743-6109.2007.00703.x

[8] Neill, S.M., Lewis, F.M., Tatnall, F.M. and Cox, N.H. (2010) British Association of Dermatologist's guideline for the menagment of lichen slerosus 2010. British Journal of Dermatology, 163, 672-682.

[9] Lagos, B.R. and Maibach, H.I. (1998) Frequency of application of topical corticosteroids: An overview. British Journal of Dermatology, 139, 763-766. http://dx.doi.org/10.1046/j.1365-2133.1998.02498.x

[10] Ya-Xian, Z., Suetake, T. and Tagami, H. (1999) Number of cell layers of the stratum corneum in normal skinRelation-ship to the anatomical location on the body, age, sex and physical parameters. Archives of Dermatological Research, 291, 555-559. http://dx.doi.org/10.1007/s004030050453 